\title{
Prevalence and predictors of vitamin D deficiency in a nationally representative sample of adults participating in the 2011-2013 Australian Health Survey
}

\author{
Eva Malacova ${ }^{1,2}$, Peihua (Rachel) Cheang ${ }^{1}$, Eleanor Dunlop ${ }^{1}$, Jill L. Sherriff ${ }^{1}$, Robyn M. Lucas ${ }^{3,4}$, \\ Robin M. Daly ${ }^{5}$, Caryl A. Nowson ${ }^{5}$ and Lucinda J. Black ${ }^{1 *}$ \\ ${ }^{1}$ School of Public Health, Curtin University, Kent Street, Bentley, WA 6102, Australia \\ ${ }^{2}$ Centre for Health Services Research, School of Population and Global Health, The University of Western Australia, 35 Stirling \\ Highway, Crawley, WA 6009, Australia \\ ${ }^{3}$ National Centre for Epidemiology and Population Health, Research School of Population Health, Australian National \\ University, Acton, ACT 2600, Australia \\ ${ }^{4}$ Centre for Ophthalmology and Visual Science, The University of Western Australia, 35 Stirling Highway, Crawley, \\ WA 6009, Australia \\ ${ }^{5}$ Institute for Physical Activity and Nutrition (IPAN), School of Exercise and Nutrition Sciences, Deakin University (Burwood \\ Campus), 221 Burwood Highway, Burwood, VIC 3125, Australia
}

(Submitted 20 July 2018 - Final revision received 4 January 2019 - Accepted 9 January 2019 - First published online 24 January 2019)

\section{Abstract}

Vitamin D deficiency is recognised as a public health problem globally, and a high prevalence of deficiency has previously been reported in Australia. This study details the prevalence of vitamin D deficiency in a nationally representative sample of Australian adults aged $\geq 25$ years, using an internationally standardised method to measure serum 25-hydroxyvitamin D (25(OH)D) concentrations and identifies demographic and lifestyle factors associated with vitamin D deficiency. We used data from the 2011-2013 Australian Health Survey ( $n 5034$ with complete information on potential predictors and serum 25(OH)D concentrations). Serum 25(OH)D concentrations were measured by a liquid chromatography-tandem MS that is certified to the reference measurement procedures developed by the National Institute of Standards and Technology, Ghent University and the US Centers for Disease Control and Prevention. Vitamin D deficiency and insufficiency were defined as serum $25(\mathrm{OH}) \mathrm{D}$ concentrations $<50 \mathrm{nmol} / \mathrm{l}$ and 50 to $<75 \mathrm{nmol} / \mathrm{l}$, respectively. Overall, $20 \%$ of participants (19\% men; $21 \% \mathrm{women})$ were classified as vitamin D deficient, with a further $43 \%$ classified as insufficient ( $45 \%$ men; $42 \%$ women). Independent predictors of vitamin D deficiency included being born in a country other than Australia or the main English-speaking countries, residing in southern (higher latitude) states of Australia, being assessed during winter or spring, being obese, smoking (women only), having low physical activity levels and not taking vitamin D or Ca supplements. Given our increasingly indoor lifestyles, there is a need to develop and promote strategies to maintain adequate vitamin D status through safe sun exposure and dietary approaches.

Key words: Vitamin D deficiency: 25-Hydroxyvitamin D: Australian Health Survey

Vitamin D deficiency is a recognised public health problem worldwide. However, the lack of international consensus on the definition of vitamin $\mathrm{D}$ deficiency and differences in the method used to measure serum 25-hydroxyvitamin D (25(OH)D) concentrations hamper comparisons across countries. Various population-based studies in the USA, New Zealand and Canada have estimated that $30-48 \%$ of adults are vitamin D deficient (defined as circulating $25(\mathrm{OH}) \mathrm{D}$ concentrations $<50 \mathrm{nmol} / \mathrm{l})^{(1-3)}$. In Europe, vitamin D deficiency (defined as $25(\mathrm{OH}) \mathrm{D}<30 \mathrm{nmol} / \mathrm{l}$ ) ranges from 9 to $24 \%$ in adults and from 1 to $8 \%$ in older adults aged $>61$ years $^{(3,4)}$. Dietary intakes of vitamin D are generally low worldwide ${ }^{(5-7)}$; indeed, the UK's Scientific Advisory Committee on Nutrition is now recommending a daily supplement containing $10 \mu \mathrm{g}$ of vitamin D in autumn and winter in order to maintain a satisfactory vitamin D status throughout the year ${ }^{(8)}$.

In a nationally representative sample of adults aged $\geq 25$ years ( $n$ 11218) participating in the 1999-2000 Australian

Abbreviations: 25(OH)D, 25-hydroxyvitamin D; AHS, Australian Health Survey; AusDiab, Australian Diabetes, Obesity and Lifestyle; LC-MS/MS, liquid chromatography-tandem MS; NHANES, National Health and Nutrition Examination Survey; NHS, National Health Survey; RMP, reference measurement procedure.

* Corresponding author: L. J. Black, email lucinda.black@curtin.edu.au 
Diabetes, Obesity and Lifestyle (AusDiab) study, 31\% were vitamin D deficient $(25(\mathrm{OH}) \mathrm{D}<50 \mathrm{nmol} / \mathrm{l})$. However, serum $25(\mathrm{OH}) \mathrm{D}$ concentrations were measured using a direct competitive chemiluminescent immunoassay (CLIA; DiaSorin Inc.) ${ }^{(9)}$. Other studies evaluating the same immunoassay have noted a negative bias compared with a liquid chromatography-tandem MS (LC-MS/MS) method that is certified to the reference measurement procedures (RMP) developed by the National Institute of Standards and Technology, Ghent University and the US Centers for Disease Control and Prevention (referred to hereafter as the RMP) ${ }^{(10,11)}$. An LC-MS/MS method certified to the RMP is widely considered the gold standard method for measuring serum $25(\mathrm{OH}) \mathrm{D}$ concentrations ${ }^{(12,13)}$. The negative bias occurs particularly at $25(\mathrm{OH}) \mathrm{D}$ concentrations above $50 \mathrm{nmol} / \mathrm{l}$, with values recorded up to $25 \mathrm{nmol} / \mathrm{l}$ below the true value ${ }^{(14,15)}$. This bias can result in misclassification of vitamin D deficiency, with more samples classified as deficient than if analysed using a method certified to the RMP. Use of a CLIA for serum $25(\mathrm{OH})$

$\mathrm{D}$ concentrations has been shown to result in up to one third of Australian adults being incorrectly classified as vitamin D deficient in comparison with an LC-MS/MS method certified to the $\mathrm{RMP}^{(12)}$

Previous studies in the Australian population are now more than a decade old and/or have not used an assay certified to the RMP to measure serum 25(OH)D concentrations. The 20112013 Australian Health Survey (AHS) is the first large, nationally representative survey in Australia to quantify serum 25(OH)D concentrations using an LC-MS/MS method certified to the $\mathrm{RMP}^{(16)}$. Using data from the AHS, we investigated the prevalence of vitamin $\mathrm{D}$ deficiency and identified its predictors in Australian adults. We limited the analysis to adults aged $\geq 25$ years in order to compare the predictors of vitamin D deficiency from the AHS with those from the 1999-2000 AusDiab study.

\section{Methods}

\section{Study design and population}

The 2011-2013 AHS comprises the National Health Survey (NHS), the National Nutrition and Physical Activity Survey (NNPAS) and the National Health Measures Survey (NHMS). Detailed methodology of the AHS can be found elsewhere ${ }^{(17)}$. The AHS is based on a stratified, multistage area sample of private households. All households were assigned analytic weights to account for their sampling probability of being included in the survey. All individuals participating in the AHS provided core information (e.g. general household and demographic data, self-reported health, smoking status and information on selected health conditions); BMI was calculated from measured weight $(\mathrm{kg})$ and height $(\mathrm{m})$ (weight/height ${ }^{2}$ ). Participants were then randomly allocated to the NHS ( $n$ 20 425) or NNPAS ( $n$ 12153). The NHS collected comprehensive health and medical information, while the NNPAS collected detailed data relating to diet and physical activity. Face-to-face interviews with participants were conducted in urban and rural areas across all states and territories of Australia. Excluded were residents of non-private dwellings, such as hostels or visitors to private dwellings. For those who were aged $>11$ years and agreed to participate in the NHMS component ( $n$ 10401), qualified phlebotomists collected blood samples for measurement of biomarkers, including serum 25(OH)D concentrations $^{(18)}$.

The present study includes data on adults aged $\geq 25$ years who participated in the NHS, provided a blood sample for measurement of serum 25(OH)D concentrations and had complete data for all potential predictors investigated. A total of 14248 adults aged $\geq 25$ years participated in the NHS component of the AHS. Of these, 8839 participants did not participate in the NHMS and, hence, did not have data for serum 25(OH)D concentrations. A further 375 participants had missing data for core variables (BMI, education level and exercise), leaving 5034 participants for the present analysis (45\% men, 55\% women)

The study was conducted in accordance with the Declaration of Helsinki. The interview components of the AHS were conducted under the Census and Statistics Act 1905. For the NHMS component, the Australian Government Department of Health and Ageing's Departmental Ethics Committee provided ethics approval in February 2011 and written informed consent was obtained from participants.

\section{Serum 25-hydroxyvitamin D concentrations}

The AHS is one of eight participating national surveys in the Vitamin D Standardization Program (VDSP) which aims to standardise measurements of serum $25(\mathrm{OH}) \mathrm{D}$ concentrations in health surveys internationally ${ }^{(10)}$. Blood samples (fasting was not required) were stored at $-80^{\circ} \mathrm{C}$ before analysis using an LC-MS/MS method that is certified to the RMP (Douglass Hanly Moir Pathology). Total vitamin D deficiency was defined as serum 25(OH)D concentrations $<50 \mathrm{nmol} / \mathrm{l}$, insufficiency as 25(OH)D 50 to $<75 \mathrm{nmol} / \mathrm{l}$ and sufficiency as $25(\mathrm{OH}) \mathrm{D} \geq 75 \mathrm{nmol} / \mathrm{l}^{(19)}$.

\section{Potential predictors of vitamin D deficiency}

We categorised age as 25-34, 35-44, 45-54, 55-64, 65-74 or $\geq 75$ years. Region of birth was described as 'Australia', 'Main English-speaking countries' (USA, Canada, UK, Republic of Ireland, New Zealand and South Africa) or 'other'. State/territory recruitment sites were New South Wales $\left(32^{\circ} \mathrm{S}\right)$, Victoria $\left(37^{\circ} \mathrm{S}\right)$, Queensland $\left(21^{\circ} \mathrm{S}\right)$, South Australia $\left(30^{\circ} \mathrm{S}\right)$, Western Australia $\left(26^{\circ} \mathrm{S}\right)$, Tasmania $\left(42^{\circ} \mathrm{S}\right)$, Northern Territory $\left(19^{\circ} \mathrm{S}\right)$ and Australian Capital Territory $\left(35^{\circ} \mathrm{S}\right)$. The Australian Bureau of Statistics (ABS) categorised region of residence according to the Accessibility/Remoteness Index of Australia, which is mapped to the census collection districts (CD) from the Census of Population and Housing. Only urban and rural areas were included in the AHS; however, the exclusion of remote and very remote areas had minimal impact on the national estimates. Area remoteness was classified based on the CD of participant's residence: $\mathrm{CD}$ with index values ranging from 0 to $0 \cdot 20$ were considered as 'major cities of Australia', while CD with index values from 0.21 to 2.40 and from 2.41 to 5.92 were classified as 'inner regional Australia' and 'other', respectively.

The 2011 Index of Relative Socioeconomic Disadvantage (IRSD) was used to describe the socio-economic status. The 
IRSD is a general socio-economic index that summarises information relating to the social and economic resources of people and households within an area, with scores ranging from low to high. A low score indicates relatively greater overall disadvantage. Scores were categorised into quintiles. Educational attainment was described as 'High/primary/no school' (high school or less), 'Certificate/diploma' (technical and further education) or 'University'. Smoking status was defined as 'current smoker' or 'ex-/non-smoker'. BMI was categorised as underweight $\left(<18.5 \mathrm{~kg} / \mathrm{m}^{2}\right)$, healthy weight $\left(18.5-24.9 \mathrm{~kg} / \mathrm{m}^{2}\right)$, overweight $\left(25 \cdot 0-29.9 \mathrm{~kg} / \mathrm{m}^{2}\right)$ or obese $\left(\geq 30.0 \mathrm{~kg} / \mathrm{m}^{2}\right)^{(20)}$. Due to small numbers of underweight participants, we grouped underweight and healthy weight together. Month of blood collection was categorised by season: 'spring (SeptemberNovember)', 'summer (December-February)', 'autumn (MarchMay)' or 'winter (June-August)'.

The ABS classified physical activity as 'low/sedentary', 'moderate' or 'high' based on the level of physical activity performed for fitness, sport or recreation and walking for transport in the past week $^{(17)}$. The data items, namely, total minutes undertaken for moderate physical activity, total minutes undertaken for vigorous physical activity, total minutes walked for fitness, recreation or sport and total minutes spent walking for transport, contributed to each category. Each physical activity category was given an intensity factor score: walking for fitness or transport $=3 \cdot 5$, moderate exercise $/$ physical activity $=5 \cdot 0$ and vigorous exercise $/$ physical activity $=7 \cdot 5$, which was subsequently multiplied by the physical activity duration.

Information on vitamin $\mathrm{D}$ and/or Ca supplement use in the last 2 weeks was collected by interview. For the purposes of this study, participants who reported using a single vitamin $\mathrm{D}$ or $\mathrm{Ca}$ supplement were considered vitamin D and/or Ca supplement users. We included Ca supplements because the majority of $\mathrm{Ca}$ supplements in Australia contain vitamin D.

\section{Statistical analyses}

Survey-weighted characteristics of participants, stratified by sex, were summarised using numbers and proportions. We reported prevalence of total vitamin D deficiency $(25(\mathrm{OH}) \mathrm{D}<50 \mathrm{nmol} / \mathrm{l})$, insufficiency $(25(\mathrm{OH}) \mathrm{D} 50$ to $<75 \mathrm{nmol} / \mathrm{l})$ and sufficiency ( $25(\mathrm{OH}) \mathrm{D} \geq 75 \mathrm{nmol} / \mathrm{l})$, stratified by sex and age group. We also reported mean serum $25(\mathrm{OH}) \mathrm{D}$ concentrations by sex and month of blood collection. All were weighted to the Australian population in 2011-2013 ${ }^{(18)}$.

Survey-weighted logistic regression models were used to determine the independent predictors of vitamin $\mathrm{D}$ deficiency $(25(\mathrm{OH}) \mathrm{D}<50 \mathrm{nmol} / \mathrm{l})$ in men and women. We stratified by sex in order to compare predictors with the AusDiab study findings. All variables were entered into the same regression models to provide adjusted OR. The model accounted for the stratification and clustering of the complex sample design using the Taylor series linearisation method ${ }^{(17)}$. All households were weighted using analytic weights provided by the AHS survey. All analyses were conducted using SAS version 9.4 (SAS Institute).

\section{Results}

Most (70\%) participants were born in Australia, with the majority (approximately 70\%) residing in New South Wales, Victoria and Queensland, and living in major cities (67\%) (Table 1). About $70 \%$ of men and $60 \%$ of women were either overweight or obese, with correspondingly low levels of physical activity ( $56 \%$ of men, $61 \%$ of women). About $13 \%$ of men and women were current smokers. Three times as many women (16\%) as men (5\%) used vitamin D and/or Ca supplements (Table 1 ).

In total, $20 \%$ of participants (19\% men, $21 \%$ women) were classified as vitamin D deficient, with a further $43 \%$ classified as insufficient ( $45 \%$ men, $42 \%$ women) (Tables 2 and 3). The highest prevalence of vitamin D deficiency (32\% men, 26\% women) was in the youngest age group (25-34 years) (Table 2). The prevalence of deficiency decreased with increasing age in men, and to a lesser extent in women, until the age of 65 years, after which there was a plateau in men and an increase in women (Table 3 and Fig. 1). Similar findings were observed for the prevalence of vitamin $\mathrm{D}$ insufficiency. The highest prevalence of severe deficiency $(25(\mathrm{OH}) \mathrm{D}<30 \mathrm{nmol} / \mathrm{l})$ was in those born in a country other than Australia or the main Englishspeaking countries ( $13 \%$ for men and $11 \%$ for women) (Table 3). The mean serum $25(\mathrm{OH}) \mathrm{D}$ concentrations were lower in winter-spring (August-October) than in summerautumn (February-March), at 55-60 and 70-75 nmol/1, respectively (Fig. 2).

Adjusted logistic regression models identified several factors significantly associated with increased odds of vitamin D deficiency (Table 4). In men only, older age was significantly associated with lower risk of vitamin D deficiency: those aged 55 years and over had approximately half the odds of vitamin D deficiency compared with 25- to 34-year-olds. Participants born in a country other than Australia and the main English-speaking countries were more than three times more likely to be vitamin D deficient than those born in Australia. As expected, those residing in southern (higher latitude) states, such as Tasmania and to a lesser extent Victoria, were more likely to be vitamin D deficient than those residing in New South Wales. Those residing in Queensland and Western Australia were less likely to be vitamin D deficient than those residing in New South Wales. Seasonal differences in vitamin D deficiency were also observed, with participants sampled in winter or spring having approximately three times the odds of vitamin D deficiency compared with those sampled in summer. Other factors associated with increased odds of vitamin D deficiency included being obese compared with having a healthy BMI; being a current smoker compared with being an ex-/non-smoker (women only); having a university education compared with high/primary/no school; having low or sedentary physical activity compared with high physical activity. Living in inner regional or 'other' areas (compared with living in major cities) was associated with a lower risk of vitamin D deficiency, as was use of vitamin D or Ca supplements. Socio-economic status (as measured by the area-level IRSD) was not associated with vitamin D deficiency. 
Table 1. Descriptive characteristics of Australian adults aged $\geq 25$ years $(n 5034)$ included in the present study (Percentages and $95 \%$ confidence intervals)

\begin{tabular}{|c|c|c|c|c|}
\hline \multirow[b]{2}{*}{ Characteristics } & \multicolumn{2}{|c|}{ Men (n 2267) } & \multicolumn{2}{|c|}{ Women (n 2767) } \\
\hline & $\%$ & $95 \% \mathrm{Cl}^{*}$ & $\%$ & $95 \% \mathrm{Cl}^{*}$ \\
\hline \multicolumn{5}{|l|}{ Age categories (years) } \\
\hline $25-34$ & $11 \cdot 8$ & $10 \cdot 2,13 \cdot 3$ & 13.5 & $11 \cdot 9,15 \cdot 0$ \\
\hline $35-44$ & $16 \cdot 2$ & $14.4,18.0$ & $17 \cdot 4$ & $15 \cdot 7,19 \cdot 1$ \\
\hline $45-54$ & $21 \cdot 2$ & $19 \cdot 1,23 \cdot 3$ & $22 \cdot 4$ & $20 \cdot 5,24 \cdot 3$ \\
\hline $55-64$ & $23 \cdot 1$ & $21 \cdot 1,25 \cdot 2$ & $21 \cdot 8$ & $20 \cdot 0,23 \cdot 7$ \\
\hline $65-74$ & $16 \cdot 7$ & $14.9,18.5$ & $16 \cdot 6$ & $14.9,18 \cdot 2$ \\
\hline$\geq 75$ & $11 \cdot 0$ & $9 \cdot 6,12 \cdot 5$ & $8 \cdot 3$ & $7 \cdot 1,9.5$ \\
\hline \multicolumn{5}{|l|}{ Region of birth } \\
\hline Australia & $68 \cdot 7$ & $66.6,70.9$ & 70.9 & $69.0,72.9$ \\
\hline Main English-speaking countries† & $14 \cdot 1$ & $12 \cdot 5,15 \cdot 7$ & $12 \cdot 2$ & $10 \cdot 8,13.5$ \\
\hline Other & $17 \cdot 2$ & $15 \cdot 4,19.0$ & $16 \cdot 9$ & $15 \cdot 2,18 \cdot 6$ \\
\hline \multicolumn{5}{|l|}{ State/territory } \\
\hline New South Wales & $30 \cdot 6$ & $28 \cdot 2,33 \cdot 1$ & $30 \cdot 3$ & $28 \cdot 1,32 \cdot 6$ \\
\hline Victoria & $22 \cdot 4$ & $20 \cdot 3,24 \cdot 5$ & $23 \cdot 7$ & $21 \cdot 7,25 \cdot 6$ \\
\hline Queensland & $22 \cdot 6$ & $20 \cdot 6,24 \cdot 6$ & $21 \cdot 3$ & $19 \cdot 6,23 \cdot 1$ \\
\hline South Australia & $7 \cdot 6$ & $6 \cdot 7,8 \cdot 6$ & $8 \cdot 2$ & $7 \cdot 3,9 \cdot 1$ \\
\hline Western Australia & 11.5 & $10 \cdot 3,12 \cdot 7$ & $10 \cdot 7$ & $9.6,11.7$ \\
\hline Tasmania & 2.9 & $2 \cdot 5,3 \cdot 3$ & 3.0 & $2 \cdot 7,3 \cdot 3$ \\
\hline Australian Capital Territory & 0.7 & $0.6,0.8$ & 0.7 & $0 \cdot 6,0.8$ \\
\hline Northern Territory & $1 \cdot 7$ & $1.5,1.9$ & $2 \cdot 0$ & $1 \cdot 7,2 \cdot 3$ \\
\hline \multicolumn{5}{|l|}{ Season } \\
\hline Summer (December-February) & $25 \cdot 0$ & $22 \cdot 9,21 \cdot 1$ & $25 \cdot 5$ & $23 \cdot 5,27 \cdot 4$ \\
\hline Autumn (March-May) & 35.5 & $33 \cdot 2,37 \cdot 8$ & 35.5 & $33 \cdot 4,37.6$ \\
\hline Winter (June-August) & $23 \cdot 7$ & $21 \cdot 7,25 \cdot 8$ & $21 \cdot 8$ & $20 \cdot 0,23 \cdot 6$ \\
\hline Spring (September-November) & $15 \cdot 7$ & $14.0,17.5$ & $17 \cdot 2$ & $15.5,19.0$ \\
\hline \multicolumn{5}{|l|}{ Remoteness areał } \\
\hline Major cities & $66 \cdot 1$ & $63.9,68.4$ & $67 \cdot 4$ & $65.4,69.5$ \\
\hline Inner regional & $24 \cdot 0$ & $21 \cdot 9,26 \cdot 1$ & $22 \cdot 4$ & $20 \cdot 5,24 \cdot 2$ \\
\hline Other & $9 \cdot 8$ & $8.5,11 \cdot 1$ & $10 \cdot 2$ & $9 \cdot 0,11 \cdot 4$ \\
\hline \multicolumn{5}{|l|}{ BMI§ } \\
\hline Healthy weight & $24 \cdot 3$ & $22 \cdot 4,26 \cdot 6$ & $39 \cdot 0$ & $37 \cdot 0,41 \cdot 4$ \\
\hline Overweight & 44.5 & $41 \cdot 4,46 \cdot 2$ & 30.7 & $28.8,32.9$ \\
\hline Obese & $31 \cdot 2$ & $29.4,33.9$ & $30 \cdot 3$ & $27.9,31.9$ \\
\hline \multicolumn{5}{|l|}{ Smoking } \\
\hline Ex-/non-smoker & $86 \cdot 9$ & $85 \cdot 3,88 \cdot 6$ & $86 \cdot 7$ & $85 \cdot 2,88 \cdot 2$ \\
\hline Current & $13 \cdot 1$ & $11 \cdot 4,14 \cdot 7$ & $13 \cdot 3$ & $11 \cdot 8,14.8$ \\
\hline \multicolumn{5}{|l|}{ Education } \\
\hline High/primary/no school & $33 \cdot 2$ & $31 \cdot 0,35 \cdot 5$ & $39 \cdot 2$ & $37 \cdot 1,41.4$ \\
\hline Certificate/diploma & $42 \cdot 4$ & $40 \cdot 0,44 \cdot 8$ & $32 \cdot 6$ & $30 \cdot 5,34 \cdot 7$ \\
\hline University & $24 \cdot 4$ & $22 \cdot 3,26 \cdot 4$ & $28 \cdot 2$ & $26 \cdot 2,30 \cdot 2$ \\
\hline \multicolumn{5}{|l|}{ Physical activityll } \\
\hline High & $13 \cdot 4$ & $11 \cdot 7,15 \cdot 0$ & $8 \cdot 8$ & $7 \cdot 5,10 \cdot 0$ \\
\hline Moderate & $30 \cdot 9$ & $28 \cdot 7,33 \cdot 2$ & 30.5 & $28 \cdot 4,32 \cdot 5$ \\
\hline Low/sedentary & $55 \cdot 7$ & $52 \cdot 3,58 \cdot 1$ & $60 \cdot 8$ & $58 \cdot 6,62.9$ \\
\hline \multicolumn{5}{|l|}{ Socio-economic status $\mathbb{\|}$} \\
\hline Lowest quintile & $18 \cdot 2$ & $16 \cdot 3,20 \cdot 1$ & $18 \cdot 3$ & $16 \cdot 6,20 \cdot 0$ \\
\hline Second quintile & 20.5 & $18 \cdot 5,22 \cdot 5$ & $19 \cdot 9$ & $18 \cdot 1,20 \cdot 6$ \\
\hline Third quintile & $20 \cdot 3$ & $18 \cdot 3,22 \cdot 2$ & $21 \cdot 8$ & $20 \cdot 0,23.6$ \\
\hline Fourth quintile & $20 \cdot 2$ & $18 \cdot 3,22 \cdot 1$ & $20 \cdot 8$ & $19 \cdot 0,22 \cdot 5$ \\
\hline Highest quintile & $20 \cdot 8$ & $18 \cdot 9,22 \cdot 7$ & $19 \cdot 3$ & $17 \cdot 6,20 \cdot 9$ \\
\hline \multicolumn{5}{|l|}{ Vitamin D or Ca supplement use } \\
\hline Yes & $5 \cdot 1$ & $4 \cdot 1,6 \cdot 2$ & $16 \cdot 0$ & $14 \cdot 4,17 \cdot 7$ \\
\hline No & $94 \cdot 9$ & $93.8,95.9$ & $84 \cdot 0$ & $82 \cdot 3,85 \cdot 6$ \\
\hline
\end{tabular}

$\mathrm{CD}$, collection districts.

* Percentages and $95 \% \mathrm{Cl}$ weighted to the Australian population in 2011-2013.

† Main English-speaking countries: USA, Canada, UK, Republic of Ireland, New Zealand and South Africa.

¥ Categorised according to the Accessibility/Remoteness Index of Australia, which is mapped to the census CD from the Census of Population and Housing (major cities: $C D$ with index values 0-0.20; inner regional: 0.21-2.40; other: 2.41-5.92).

$\S$ Healthy weight $\left(18.5-24.9 \mathrm{~kg} / \mathrm{m}^{2}\right.$; this group includes a small number of underweight participants $\left.<18.5 \mathrm{~kg} / \mathrm{m}^{2}\right)$, overweight $\left(25 \cdot 0-29.9 \mathrm{~kg} / \mathrm{m}^{2}\right)$ and obese $\left(\geq 30.0 \mathrm{~kg} / \mathrm{m}^{2}\right)$.

II Based on the level of physical activity performed for fitness, sport or recreation and walking for transport in the past week.

Il Based on 2011 Index of Relative Socioeconomic Disadvantage. 
Table 2. Survey-weighted prevalence of serum 25 -hydroxyvitamin D concentrations $<30,<50$ and $<75 \mathrm{nmol} / \mathrm{l}$ in Australian adults aged $\geq 25$ years ( $n 5034$ ) included in the present study

(Percentages and 95\% confidence intervals)

\begin{tabular}{|c|c|c|c|c|c|c|c|}
\hline \multirow[b]{2}{*}{ Characteristics } & \multirow[b]{2}{*}{$n$} & \multicolumn{2}{|c|}{$<30 \mathrm{nmol} / \mathrm{l}$} & \multicolumn{2}{|c|}{$<50 \mathrm{nmol} / \mathrm{l}$} & \multicolumn{2}{|c|}{$<75 \mathrm{nmol} / \mathrm{l}$} \\
\hline & & $\%$ & $95 \% \mathrm{Cl}^{*}$ & $\%$ & $95 \% \mathrm{Cl}^{*}$ & $\%$ & $95 \% \mathrm{Cl}^{*}$ \\
\hline All & 5034 & 4.5 & $3 \cdot 8,5 \cdot 2$ & $20 \cdot 1$ & $18 \cdot 8,21 \cdot 4$ & 63.4 & $61 \cdot 8,65 \cdot 0$ \\
\hline \multicolumn{8}{|l|}{ Age categories (years) } \\
\hline $25-34$ & 669 & $8 \cdot 8$ & $6.0,11.5$ & $28 \cdot 8$ & $24 \cdot 5,33 \cdot 0$ & $68 \cdot 3$ & $64 \cdot 1,72 \cdot 6$ \\
\hline $35-44$ & 884 & $7 \cdot 4$ & $5 \cdot 2,9 \cdot 6$ & $23 \cdot 1$ & $19 \cdot 7,26 \cdot 4$ & $66 \cdot 7$ & $63.0,70.4$ \\
\hline $45-54$ & 1059 & $4 \cdot 0$ & $2 \cdot 6,5 \cdot 4$ & 20.7 & $17 \cdot 8,23 \cdot 7$ & $66 \cdot 7$ & $63 \cdot 2,70 \cdot 1$ \\
\hline $55-64$ & 1131 & 1.9 & $0.9,2.8$ & $16 \cdot 8$ & $14 \cdot 2,19 \cdot 3$ & $61 \cdot 2$ & $57 \cdot 9,64 \cdot 6$ \\
\hline $65-74$ & 813 & 2.5 & $1 \cdot 3,3 \cdot 8$ & $15 \cdot 1$ & $12 \cdot 3,17.9$ & $56 \cdot 9$ & $53 \cdot 0,60.9$ \\
\hline$\geq 75$ & 478 & $4 \cdot 3$ & $2 \cdot 1,6 \cdot 5$ & 18.5 & $14 \cdot 5,22 \cdot 5$ & 59.9 & $54 \cdot 9,65 \cdot 0$ \\
\hline \multicolumn{8}{|l|}{ Region of birth } \\
\hline Australia & 3567 & $2 \cdot 6$ & $2 \cdot 0,3 \cdot 2$ & $16 \cdot 2$ & $14 \cdot 8,17 \cdot 7$ & $60 \cdot 0$ & $58 \cdot 1,62 \cdot 0$ \\
\hline Main English-speaking countries† & 667 & $4 \cdot 3$ & $2 \cdot 5,6 \cdot 1$ & $16 \cdot 1$ & $12 \cdot 8,19 \cdot 3$ & $58 \cdot 0$ & $53 \cdot 7,62 \cdot 4$ \\
\hline Other & 800 & $12 \cdot 1$ & $9 \cdot 4,14.8$ & 38.4 & $34 \cdot 5,42 \cdot 3$ & 80.6 & $77.5,83.8$ \\
\hline \multicolumn{8}{|l|}{ State/territory } \\
\hline New South Wales & 824 & $5 \cdot 4$ & $3.9,7 \cdot 0$ & $20 \cdot 1$ & $17 \cdot 3,22 \cdot 9$ & $61 \cdot 3$ & $57 \cdot 9,64 \cdot 7$ \\
\hline Victoria & 734 & $6 \cdot 2$ & $4.5,8.0$ & $29 \cdot 0$ & $25 \cdot 7,32 \cdot 4$ & 71.6 & $68 \cdot 3,74.9$ \\
\hline Queensland & 848 & $1 \cdot 8$ & $0.9,2.7$ & $10 \cdot 2$ & $8 \cdot 1,12 \cdot 2$ & $54 \cdot 1$ & $50 \cdot 7,57 \cdot 5$ \\
\hline South Australia & 626 & $5 \cdot 0$ & $3 \cdot 3,6 \cdot 7$ & $24 \cdot 8$ & $21 \cdot 3,28 \cdot 3$ & $74 \cdot 8$ & $71 \cdot 3,78 \cdot 3$ \\
\hline Western Australia & 719 & 2.7 & $1.5,3.9$ & $15 \cdot 8$ & $13 \cdot 1,18 \cdot 5$ & $60 \cdot 7$ & $57 \cdot 1,64 \cdot 3$ \\
\hline Tasmania & 544 & $5 \cdot 5$ & $3 \cdot 5,7 \cdot 6$ & $26 \cdot 2$ & $22 \cdot 3,30 \cdot 1$ & $68 \cdot 0$ & $63 \cdot 8,72 \cdot 1$ \\
\hline Australian Capital Territory & 271 & 0.6 & $0.0,1.4$ & 9.4 & $5.4,13.4$ & $55 \cdot 3$ & $48 \cdot 5,62 \cdot 0$ \\
\hline Northern Territory & 468 & $7 \cdot 1$ & $2 \cdot 4,11 \cdot 8$ & 25.7 & $20.5,30.9$ & $69 \cdot 0$ & $64.6,73.5$ \\
\hline \multicolumn{8}{|l|}{ Season } \\
\hline Summer (December-February) & 1292 & $2 \cdot 3$ & $1 \cdot 3,3 \cdot 3$ & 13.9 & $11 \cdot 6,16 \cdot 2$ & 59.9 & $56 \cdot 7,63 \cdot 1$ \\
\hline Winter (June-August) & 1168 & $8 \cdot 9$ & $6 \cdot 9,10.8$ & 31.5 & $28 \cdot 3,34 \cdot 6$ & 70.4 & $67 \cdot 3,73 \cdot 5$ \\
\hline Autumn (March-May) & 1786 & $2 \cdot 6$ & $1 \cdot 7,3 \cdot 5$ & $13 \cdot 8$ & $11 \cdot 9,15 \cdot 7$ & $57 \cdot 1$ & $54 \cdot 3,59 \cdot 8$ \\
\hline Spring (September-November) & 788 & 5.9 & $3 \cdot 9,8 \cdot 0$ & $27 \cdot 4$ & $23 \cdot 7,31 \cdot 1$ & $72 \cdot 8$ & $69 \cdot 2,76 \cdot 5$ \\
\hline \multicolumn{8}{|l|}{ Remoteness areał } \\
\hline Major cities & 3133 & $5 \cdot 8$ & $4 \cdot 8,6 \cdot 7$ & 23.8 & $22 \cdot 1,25 \cdot 5$ & $65 \cdot 7$ & $63.8,67.6$ \\
\hline Inner regional & 1082 & $2 \cdot 1$ & $1 \cdot 1,3 \cdot 1$ & $12 \cdot 2$ & $10 \cdot 8,15 \cdot 5$ & 59.5 & $56.0,62.9$ \\
\hline Other & 819 & 1.5 & $0.6,2.5$ & $11 \cdot 3$ & $8 \cdot 6,14 \cdot 0$ & $57 \cdot 2$ & $52 \cdot 5,61.8$ \\
\hline \multicolumn{8}{|l|}{ BMI§ } \\
\hline Healthy weight & 1631 & 4.9 & $3 \cdot 6,6 \cdot 2$ & $19 \cdot 2$ & $16 \cdot 9,21 \cdot 6$ & $57 \cdot 7$ & $54 \cdot 8,60 \cdot 5$ \\
\hline Overweight & 1858 & 3.7 & $2 \cdot 6,4 \cdot 7$ & $17 \cdot 1$ & $15 \cdot 1,19 \cdot 2$ & $61 \cdot 4$ & $58 \cdot 8,64 \cdot 1$ \\
\hline Obese & 1545 & $5 \cdot 0$ & $3 \cdot 8,6 \cdot 3$ & $24 \cdot 6$ & $22 \cdot 0,27 \cdot 1$ & $71 \cdot 8$ & $69.1,74.5$ \\
\hline \multicolumn{8}{|l|}{ Smoking } \\
\hline Ex-/non-smoker & 4366 & $4 \cdot 0$ & $3 \cdot 3,4 \cdot 7$ & $19 \cdot 3$ & $17 \cdot 9,20 \cdot 6$ & 63.6 & $61.9,65.3$ \\
\hline Current & 668 & $7 \cdot 6$ & $5 \cdot 1,10 \cdot 1$ & $25 \cdot 6$ & $21 \cdot 6,29 \cdot 6$ & $62 \cdot 1$ & $57 \cdot 6,66 \cdot 5$ \\
\hline \multicolumn{8}{|l|}{ Education } \\
\hline High/primary/no school & 1795 & 3.6 & $2 \cdot 6,4.5$ & $19 \cdot 4$ & $17 \cdot 3,21 \cdot 6$ & 61.4 & $58 \cdot 8,64.0$ \\
\hline Certificate/diploma & 1846 & $3 \cdot 0$ & $2 \cdot 1,3 \cdot 9$ & $16 \cdot 6$ & $14 \cdot 6,18 \cdot 6$ & $61 \cdot 1$ & $58 \cdot 5,63.8$ \\
\hline University & 1393 & $7 \cdot 9$ & $6 \cdot 1,9 \cdot 7$ & $26 \cdot 0$ & $23 \cdot 2,28 \cdot 8$ & $69 \cdot 4$ & $66 \cdot 4,72 \cdot 3$ \\
\hline \multicolumn{8}{|l|}{ Physical activity\|l } \\
\hline High & 569 & $2 \cdot 0$ & $0.6,3.4$ & $14 \cdot 2$ & $10 \cdot 6,17 \cdot 8$ & $57 \cdot 1$ & $52 \cdot 2,62 \cdot 1$ \\
\hline Moderate & 1562 & $3 \cdot 3$ & $2 \cdot 3,4 \cdot 4$ & $16 \cdot 3$ & $14 \cdot 1,18 \cdot 5$ & $58 \cdot 0$ & $55 \cdot 1,60 \cdot 9$ \\
\hline Low/sedentary & 2903 & $5 \cdot 6$ & $4 \cdot 6,6 \cdot 6$ & 23.2 & $21 \cdot 4,25 \cdot 0$ & $67 \cdot 4$ & $65 \cdot 4,69 \cdot 4$ \\
\hline \multicolumn{8}{|l|}{ Socio-economic status $\mathbb{1}$} \\
\hline Lowest quintile & 877 & $4 \cdot 7$ & $3 \cdot 0,6 \cdot 4$ & 21.4 & $18 \cdot 2,24 \cdot 6$ & $65 \cdot 0$ & $61 \cdot 2,68 \cdot 8$ \\
\hline Second quintile & 958 & $4 \cdot 7$ & $3 \cdot 2,6 \cdot 3$ & 20.5 & $17 \cdot 5,23.4$ & $62 \cdot 2$ & $58 \cdot 7,65 \cdot 8$ \\
\hline Third quintile & 1058 & 4.5 & $3 \cdot 0,6 \cdot 0$ & $19 \cdot 0$ & $16 \cdot 2,21 \cdot 8$ & $63 \cdot 8$ & $60 \cdot 3,67 \cdot 2$ \\
\hline Fourth quintile & 1067 & $4 \cdot 3$ & $2 \cdot 9,5 \cdot 8$ & $19 \cdot 1$ & $16 \cdot 3,21 \cdot 9$ & $64 \cdot 6$ & $61 \cdot 2,68 \cdot 0$ \\
\hline Highest quintile & 1074 & $4 \cdot 2$ & $2 \cdot 7,5 \cdot 8$ & $20 \cdot 7$ & $17 \cdot 7,23 \cdot 7$ & $61 \cdot 6$ & $58 \cdot 1,65 \cdot 1$ \\
\hline \multicolumn{8}{|l|}{ Vitamin D or Ca supplement use } \\
\hline Yes & 590 & 0.9 & $0.0,1.8$ & $7 \cdot 2$ & $4 \cdot 7,9 \cdot 6$ & $45 \cdot 2$ & $40 \cdot 4,50 \cdot 1$ \\
\hline No & 4444 & 4.9 & $4 \cdot 2,5 \cdot 7$ & $21 \cdot 7$ & $20 \cdot 3,23 \cdot 1$ & $65 \cdot 7$ & $64.0,67 \cdot 3$ \\
\hline
\end{tabular}

$\mathrm{CD}$, collection districts.

* Percentages weighted to the Australian population in 2011-2013.

† Main English-speaking countries: USA, Canada, UK, Republic of Ireland, New Zealand and South Africa.

$¥$ Categorised according to the Accessibility/Remoteness Index of Australia, which is mapped to the census CD from the Census of Population and Housing (major cities: CD with index values 0-0.20; inner regional: 0.21-2.40; other: 2.41-5.92).

$\S$ Healthy weight $\left(18.5-24.9 \mathrm{~kg} / \mathrm{m}^{2}\right.$; this group includes a small number of underweight participants $\left.<18.5 \mathrm{~kg} / \mathrm{m}^{2}\right)$, overweight $\left(25 \cdot 0-29.9 \mathrm{~kg} / \mathrm{m}^{2}\right)$ and obese $\left(\geq 30.0 \mathrm{~kg} / \mathrm{m}^{2}\right)$.

II Based on the level of physical activity performed for fitness, sport or recreation and walking for transport in the past week.

I Based on 2011 Index of Relative Socioeconomic Disadvantage. 
Table 3. Survey-weighted prevalence of serum 25-hydroxyvitamin D concentrations $<30,<50$ and $<75 \mathrm{nmol} / \mathrm{l}$ in Australian adults aged $\geq 25$ years included in the present study, stratified by sex (men, 2267; women, $n$ 2767)

(Percentages and $95 \%$ confidence intervals)

\begin{tabular}{|c|c|c|c|c|c|c|c|c|c|c|c|c|c|c|}
\hline \multirow[b]{3}{*}{ Characteristics } & \multicolumn{7}{|c|}{ Men } & \multicolumn{7}{|c|}{ Women } \\
\hline & \multirow[b]{2}{*}{$n$} & \multicolumn{2}{|c|}{$<30 \mathrm{nmol} / \mathrm{l}$} & \multicolumn{2}{|c|}{$<50 \mathrm{nmol} / \mathrm{l}$} & \multicolumn{2}{|c|}{$<75 \mathrm{nmol} / \mathrm{l}$} & \multirow[b]{2}{*}{$n$} & \multicolumn{2}{|c|}{$<30 \mathrm{nmol} / \mathrm{l}$} & \multicolumn{2}{|c|}{$<50 \mathrm{nmol} / \mathrm{l}$} & \multicolumn{2}{|c|}{$<75 \mathrm{nmol} / \mathrm{l}$} \\
\hline & & $\%$ & $95 \% \mathrm{Cl}^{\star}$ & $\%$ & $95 \% \mathrm{Cl}^{*}$ & $\%$ & $95 \% \mathrm{Cl}^{*}$ & & $\%$ & $95 \% \mathrm{Cl}^{*}$ & $\%$ & $95 \% \mathrm{Cl}^{*}$ & $\%$ & $95 \% \mathrm{Cl}^{*}$ \\
\hline \multicolumn{7}{|l|}{ Age categories (years) } & $61 \cdot 7,66 \cdot 3$ & 2767 & 4.9 & $3 \cdot 9,5 \cdot 8$ & $20 \cdot 9$ & $19 \cdot 0,22 \cdot 7$ & $62 \cdot 9$ & $60 \cdot 8,65 \cdot 1$ \\
\hline $25-34$ & 272 & $9 \cdot 7$ & $5 \cdot 4,14 \cdot 1$ & 32.5 & $25 \cdot 8,39 \cdot 2$ & 71.9 & $65 \cdot 6,78 \cdot 1$ & 397 & 8.0 & $4 \cdot 6,11 \cdot 5$ & $26 \cdot 0$ & $20 \cdot 6,31.5$ & $65 \cdot 7$ & $60 \cdot 0,71.5$ \\
\hline $35-44$ & 377 & $8 \cdot 3$ & $4.7,11.9$ & $22 \cdot 6$ & $17 \cdot 5,27 \cdot 6$ & $69 \cdot 4$ & $64 \cdot 0,74 \cdot 7$ & 507 & $6 \cdot 7$ & $4 \cdot 0,9 \cdot 4$ & $23 \cdot 4$ & $18 \cdot 9,28 \cdot 0$ & $64 \cdot 6$ & $54.5,69.7$ \\
\hline $45-54$ & 475 & 3.9 & $1 \cdot 9,5 \cdot 9$ & 21.4 & $16 \cdot 9,25 \cdot 9$ & 68.9 & $63 \cdot 8,74.0$ & 584 & $4 \cdot 1$ & $2 \cdot 1,6 \cdot 1$ & $20 \cdot 2$ & $16 \cdot 3,24 \cdot 1$ & 64.8 & $60.2,69.5$ \\
\hline $55-64$ & 526 & 1.0 & $0.0,2 \cdot 1$ & $16 \cdot 5$ & $12 \cdot 8,20 \cdot 3$ & $60 \cdot 8$ & $55 \cdot 8,65 \cdot 7$ & 605 & $2 \cdot 6$ & $1 \cdot 2,4 \cdot 1$ & $17 \cdot 0$ & $13.5,20.5$ & 61.7 & $57 \cdot 1,66 \cdot 3$ \\
\hline $65-74$ & 375 & $1 \cdot 3$ & $0 \cdot 1,2 \cdot 4$ & 11.4 & $7 \cdot 8,15 \cdot 1$ & $55 \cdot 7$ & $49 \cdot 9,61 \cdot 6$ & 438 & $3 \cdot 6$ & $1 \cdot 6,5 \cdot 6$ & $18 \cdot 2$ & $14 \cdot 0,22 \cdot 3$ & $57 \cdot 9$ & $52 \cdot 6,63 \cdot 3$ \\
\hline$\geq 75$ & 242 & 2.5 & $0.1,4.9$ & $13 \cdot 2$ & $8.6,17.9$ & $57 \cdot 4$ & $50 \cdot 3,64.5$ & 236 & $6 \cdot 3$ & $2 \cdot 6,10 \cdot 0$ & 24.4 & $17 \cdot 8,30 \cdot 9$ & $62 \cdot 8$ & $55.7,69.9$ \\
\hline \multicolumn{15}{|l|}{ Region of birth } \\
\hline Australia & 1578 & $2 \cdot 0$ & $1 \cdot 2,2 \cdot 7$ & $15 \cdot 1$ & $13 \cdot 1,17 \cdot 2$ & $60 \cdot 4$ & $57 \cdot 6,63 \cdot 3$ & 1989 & 3.2 & $2 \cdot 2,4 \cdot 1$ & $17 \cdot 1$ & $15 \cdot 1,19 \cdot 1$ & $59 \cdot 7$ & $57 \cdot 1,62 \cdot 3$ \\
\hline Main English-speaking countries $†$ & 319 & $2 \cdot 6$ & $0.8,4.4$ & $12 \cdot 0$ & $8 \cdot 0,15 \cdot 9$ & $59 \cdot 6$ & $53 \cdot 4,65 \cdot 8$ & 348 & 5.9 & $2.9,8.9$ & $19 \cdot 8$ & $14.8,24.9$ & $56 \cdot 6$ & $50 \cdot 5,62 \cdot 7$ \\
\hline Other & 370 & $13 \cdot 1$ & $8 \cdot 9,17 \cdot 2$ & 39.8 & $34.0,45.6$ & 80.6 & $76 \cdot 1,85 \cdot 2$ & 430 & 11.2 & $7 \cdot 6,14.9$ & 37.1 & $31 \cdot 7,42.5$ & $80 \cdot 6$ & $76 \cdot 2,85 \cdot 1$ \\
\hline \multicolumn{15}{|l|}{ State/territory } \\
\hline New South Wales & 382 & 4.7 & $2 \cdot 6,6 \cdot 8$ & $19 \cdot 2$ & $15 \cdot 2,23 \cdot 2$ & 61.3 & $56 \cdot 3,66 \cdot 3$ & 442 & $6 \cdot 1$ & $3.8,8.3$ & $20 \cdot 9$ & $17 \cdot 0,24 \cdot 7$ & $61 \cdot 2$ & $56 \cdot 6,65 \cdot 9$ \\
\hline Victoria & 324 & 7.4 & $4.5,10 \cdot 3$ & $30 \cdot 7$ & $25 \cdot 6,35 \cdot 8$ & 76.5 & $71 \cdot 9,81 \cdot 2$ & 410 & $5 \cdot 3$ & $3 \cdot 1,7 \cdot 5$ & $27 \cdot 7$ & $23 \cdot 3,32 \cdot 1$ & $67 \cdot 7$ & $63 \cdot 1,72 \cdot 3$ \\
\hline Queensland & 394 & 1.0 & $0.02,2.0$ & 7.5 & $4 \cdot 9,10 \cdot 1$ & 51.5 & $46 \cdot 4,56 \cdot 5$ & 454 & 2.5 & $1 \cdot 1,4.0$ & 12.5 & $9 \cdot 4,15 \cdot 7$ & $56 \cdot 5$ & $51 \cdot 8,61 \cdot 1$ \\
\hline South Australia & 272 & $2 \cdot 4$ & $0.6,4.1$ & $25 \cdot 2$ & $19 \cdot 8,30.6$ & $76 \cdot 8$ & $71.7,81.9$ & 354 & $7 \cdot 1$ & $4.3,9 \cdot 8$ & 24.5 & $20 \cdot 0,29 \cdot 1$ & $73 \cdot 3$ & $68.5,78.0$ \\
\hline Western Australia & 343 & $2 \cdot 3$ & $0.7,3.9$ & $13 \cdot 6$ & $9.9,17 \cdot 3$ & 59.7 & $54.5,65.0$ & 376 & 3.0 & $1.3,4.7$ & $17 \cdot 8$ & $13 \cdot 9,21 \cdot 7$ & 61.5 & $56 \cdot 5,66 \cdot 5$ \\
\hline Tasmania & 233 & $6 \cdot 4$ & $3.0,9.9$ & 28.5 & $22 \cdot 3,34 \cdot 6$ & 74.7 & $68 \cdot 7,80 \cdot 6$ & 311 & 4.8 & $2 \cdot 4,7 \cdot 2$ & 24.4 & $19 \cdot 4,29 \cdot 4$ & $62 \cdot 6$ & $57 \cdot 0,68 \cdot 1$ \\
\hline Australian Capital Territory & 122 & 0.5 & $0.0,1.5$ & $6 \cdot 3$ & $2 \cdot 1,10 \cdot 5$ & $55 \cdot 2$ & $45 \cdot 5,64 \cdot 8$ & 149 & 0.7 & $0.0,1 \cdot 7$ & 11.9 & $5 \cdot 6,18 \cdot 2$ & $55 \cdot 4$ & $46 \cdot 0,64 \cdot 7$ \\
\hline Northern Territory & 197 & 5.5 & $2 \cdot 1,8 \cdot 8$ & $23 \cdot 1$ & $17 \cdot 1,29 \cdot 0$ & $69 \cdot 8$ & $63 \cdot 4,76 \cdot 3$ & 271 & 8.3 & $0 \cdot 7,15 \cdot 8$ & $27 \cdot 6$ & $19.9,35 \cdot 2$ & 68.5 & $62.4,74.5$ \\
\hline \multicolumn{15}{|l|}{ Season } \\
\hline Summer (December-February) & 561 & $2 \cdot 0$ & $0.7,3.4$ & 13.5 & $10 \cdot 2,16 \cdot 8$ & $61 \cdot 7$ & $56 \cdot 9,66 \cdot 4$ & 731 & $2 \cdot 6$ & $1 \cdot 2,4 \cdot 0$ & $14 \cdot 2$ & $11 \cdot 1,17 \cdot 4$ & $58 \cdot 4$ & $54 \cdot 1,62 \cdot 7$ \\
\hline Autumn (March-May) & 819 & 1.8 & $0.6,2.9$ & $12 \cdot 0$ & $9 \cdot 3,14.7$ & $56 \cdot 1$ & $52 \cdot 1,60 \cdot 2$ & 967 & 3.3 & $1.9,4.6$ & $15 \cdot 4$ & $12 \cdot 7,18 \cdot 0$ & 57.8 & $54.1,61.5$ \\
\hline Winter (June-August) & 543 & 9.4 & $6 \cdot 4,12 \cdot 3$ & 32.5 & $27 \cdot 8,37 \cdot 1$ & 71.0 & $66 \cdot 5,75 \cdot 6$ & 625 & 8.4 & $5 \cdot 8,10 \cdot 9$ & $30 \cdot 6$ & $26 \cdot 3,34 \cdot 9$ & 69.8 & $65.5,74.1$ \\
\hline Spring (September-November) & 344 & 4.4 & $1.9,7 \cdot 0$ & 24.5 & $19 \cdot 2,29 \cdot 8$ & 74.8 & $69 \cdot 6,80 \cdot 1$ & 444 & $7 \cdot 1$ & $4 \cdot 0,10 \cdot 2$ & $29 \cdot 6$ & $24.5,34.7$ & $71 \cdot 3$ & $66 \cdot 3,76 \cdot 3$ \\
\hline \multicolumn{15}{|l|}{ Remoteness areał } \\
\hline Major cities & 1398 & $5 \cdot 8$ & $4 \cdot 3,7 \cdot 2$ & $24 \cdot 1$ & $21 \cdot 5,26 \cdot 7$ & 67.5 & $64 \cdot 7,70 \cdot 2$ & 1735 & 5.8 & $4.5,7 \cdot 1$ & 23.6 & $21 \cdot 3,25 \cdot 9$ & $64 \cdot 3$ & $61 \cdot 7,66 \cdot 9$ \\
\hline Inner regional & 499 & 0.8 & $0.3,1 \cdot 3$ & $9 \cdot 7$ & $6 \cdot 8,12 \cdot 5$ & $59 \cdot 2$ & $54 \cdot 2,64 \cdot 3$ & 583 & $3 \cdot 3$ & $1 \cdot 5,5 \cdot 1$ & $16 \cdot 3$ & $12 \cdot 7,20 \cdot 0$ & $59 \cdot 7$ & $54 \cdot 9,64.5$ \\
\hline Other & 370 & 0.5 & $0.02,1.1$ & $9 \cdot 7$ & $5 \cdot 8,13 \cdot 6$ & $52 \cdot 2$ & $45 \cdot 2,59 \cdot 2$ & 449 & $2 \cdot 4$ & $0.7,4.1$ & $12 \cdot 6$ & $8.9,16 \cdot 4$ & $61 \cdot 1$ & $54.9,67.3$ \\
\hline \multicolumn{15}{|l|}{ BMI§ } \\
\hline Healthy weight & 551 & 4.7 & $2.5,6.9$ & $19 \cdot 6$ & $15 \cdot 6,23 \cdot 6$ & $56 \cdot 9$ & $52 \cdot 0,61 \cdot 7$ & 1080 & $5 \cdot 1$ & $3 \cdot 4,6 \cdot 7$ & $19 \cdot 1$ & $16 \cdot 2,21 \cdot 9$ & $58 \cdot 1$ & $54 \cdot 6,61 \cdot 6$ \\
\hline Overweight & 1008 & 3.9 & $2 \cdot 5,5.4$ & $17 \cdot 8$ & $15 \cdot 0,20 \cdot 6$ & $62 \cdot 8$ & $59 \cdot 2,66 \cdot 3$ & 850 & 3.3 & $1.9,4 \cdot 8$ & $16 \cdot 3$ & $13 \cdot 3,19 \cdot 3$ & 59.9 & $55.9,63.8$ \\
\hline Obese & 708 & 3.7 & $2 \cdot 1,5 \cdot 3$ & $20 \cdot 8$ & $17 \cdot 4,24 \cdot 3$ & 71.2 & $67 \cdot 3,75 \cdot 1$ & 837 & $6 \cdot 2$ & $4.3,8 \cdot 1$ & 27.9 & $24 \cdot 3,31.5$ & $72 \cdot 4$ & $68 \cdot 7,76 \cdot 0$ \\
\hline \multicolumn{15}{|l|}{ Smoking } \\
\hline Ex-/non-smoker & 1963 & 3.6 & $2 \cdot 6,4.5$ & $18 \cdot 3$ & $16 \cdot 3,20 \cdot 3$ & 64.8 & $62 \cdot 3,67 \cdot 3$ & 2403 & 4.4 & $3.4,5.4$ & $20 \cdot 1$ & $18 \cdot 1,22 \cdot 0$ & $62 \cdot 6$ & $60 \cdot 3,64.9$ \\
\hline Current & 304 & 7.3 & $3 \cdot 6,11 \cdot 0$ & $25 \cdot 0$ & $19 \cdot 1,30 \cdot 8$ & 58.6 & $51 \cdot 9,65 \cdot 2$ & 364 & $7 \cdot 8$ & $4 \cdot 5,11 \cdot 1$ & $26 \cdot 1$ & $20 \cdot 7,31.5$ & 65.0 & $59.0,70.9$ \\
\hline Education & & & & & & & & & & & & & & \\
\hline High/primary/no school & 747 & $3 \cdot 0$ & $1.5,4.5$ & 17.5 & $14 \cdot 2,20 \cdot 7$ & 59.8 & $55 \cdot 7,64 \cdot 0$ & 1048 & 4.0 & $2 \cdot 7,5 \cdot 3$ & $20 \cdot 8$ & $18 \cdot 0,23 \cdot 7$ & $62 \cdot 5$ & $59.1,65.9$ \\
\hline Certificate/diploma & 939 & 2.5 & $1.3,3.8$ & $15 \cdot 5$ & $12 \cdot 8,18 \cdot 3$ & $60 \cdot 7$ & $57 \cdot 0,64 \cdot 3$ & 907 & 3.5 & $2 \cdot 1,4.9$ & $17 \cdot 7$ & $14 \cdot 7,20 \cdot 7$ & 61.7 & $57 \cdot 8,65.5$ \\
\hline University & 581 & $8 \cdot 1$ & $5.4,10.9$ & 28.0 & $23 \cdot 6,32 \cdot 3$ & $75 \cdot 5$ & $71 \cdot 3,79 \cdot 7$ & 812 & $7 \cdot 7$ & $5 \cdot 3,10 \cdot 1$ & 24.5 & $20 \cdot 8,28 \cdot 2$ & 64.9 & $60 \cdot 9,68.9$ \\
\hline Physical activity\| & & & & & & & & & & & & & & \\
\hline High & 303 & $3 \cdot 2$ & $0.7,5 \cdot 6$ & $16 \cdot 0$ & $10 \cdot 8,21 \cdot 1$ & $60 \cdot 0$ & $53 \cdot 4,66 \cdot 6$ & 266 & 0.5 & $0.0,1 \cdot 1$ & 11.9 & $7 \cdot 0,16 \cdot 8$ & 53.5 & $46 \cdot 0,61 \cdot 0$ \\
\hline Moderate & 721 & $2 \cdot 8$ & $1 \cdot 3,4.3$ & $17 \cdot 3$ & $14 \cdot 0,20 \cdot 7$ & 59.5 & $55 \cdot 2,63 \cdot 7$ & 841 & 3.8 & $2 \cdot 3,5 \cdot 3$ & $15 \cdot 4$ & $12 \cdot 5,18 \cdot 3$ & $56 \cdot 8$ & $52 \cdot 8,60 \cdot 8$ \\
\hline Low/sedentary & 1243 & $5 \cdot 0$ & $3.5,6.4$ & $21 \cdot 0$ & $18.4,23.6$ & $67 \cdot 4$ & $64.4,70.5$ & 1660 & $6 \cdot 0$ & $4 \cdot 6,7 \cdot 4$ & 24.9 & $22 \cdot 4,27 \cdot 4$ & $67 \cdot 3$ & $64 \cdot 7,70 \cdot 0$ \\
\hline
\end{tabular}




\section{Discussion}

We found that $20 \%$ of adults aged $\geq 25$ years who participated in the 2011-2013 AHS were vitamin D deficient (25(OH)D $<50 \mathrm{nmol} / \mathrm{l}$ ) ( $5 \%$ of adults had concentrations $<30 \mathrm{nmol} / \mathrm{l}$ ), with a further $43 \%$ vitamin D insufficient (25(OH)D 50 to $<75 \mathrm{nmol} / \mathrm{l})$. By comparing our AHS-based prevalence data with census population data ${ }^{(21)}$, we estimated that, in 2011, approximately 9.83 , 3.29 and 0.81 million Australian adults aged $\geq 25$ years had serum $25(\mathrm{OH}) \mathrm{D}$ concentrations $<75,<50$ and $<30 \mathrm{nmol} / \mathrm{l}$, respectively.

Approximately $30 \%$ of adults aged $\geq 25$ years who participated in the nationally representative 1999-2000 AusDiab study were vitamin D deficient; however, it is not possible to assess a trend in vitamin D deficiency over time, since the AHS used an LC-MS/MS method certified to the RMP to quantify $25(\mathrm{OH}) \mathrm{D}$ concentrations, while the AusDiab study used a DiaSorin CLIA. Another difference between the AusDiab study and the AHS was the sampling time frame, that is, the AusDiab study sampled specific regions in specific months, while the AHS sampled throughout the year.

This difficulty in comparing the prevalence of vitamin D deficiency over time due to a change in analytical methodology also arose in the USA, where the earlier National Health and Nutrition Examination Survey (NHANES) surveys (up to and including the 2000-2006 survey) used an immunoassay, but subsequent NHANES surveys used an LC-MS/MS method that was certified to the $\mathrm{RMP}^{(22)}$. An initial comparison indicated a decrease in mean serum $25(\mathrm{OH}) \mathrm{D}$ concentrations between the 1988-1994 NHANES and 2000-2006 NHANES ${ }^{(23)}$. However, the results from the two surveys were later harmonised using the VDSP protocol, a mathematical model to calibrate nonstandardised assay results to allow comparison with results of standardised assays ${ }^{(10)}$. The harmonised results indicated there was no change in the prevalence of vitamin D deficiency in the USA between 1988-1994 and 2000-2006 ${ }^{(24)}$. Serum 25(OH)D data from NHANES 2001-2010 (measured by a LC-MS/MS method certified to the RMP) showed that $30 \%$ of adults were vitamin D deficient $(25(\mathrm{OH}) \mathrm{D}<50 \mathrm{nmol} / \mathrm{l})$ and $41 \%$ were insufficient $(50 \text { to }<75 \mathrm{nmol} / \mathrm{l})^{(1)}$. Similar harmonisation procedures have been applied to data from several European studies with varying effects on the estimates of the prevalence of vitamin $D$ deficiency. ${ }^{(4)}$ In the Irish National Adult Nutrition Survey, the estimated prevalence of serum $25(\mathrm{OH}) \mathrm{D}<30 \mathrm{nmol} / \mathrm{l}$ was $6.5 \%$. After harmonisation, the 'true' prevalence of deficiency was estimated as $11.4 \%{ }^{(25)}$. In contrast, in the German Health Interview and Examination Survey for Adults, harmonisation decreased the prevalence estimate from 28.0 to $12 \cdot 9 \%^{(4)}$.

Most predictors of vitamin D deficiency were similar between the AusDiab study and the AHS, including low physical activity, obesity, residing in the southern part of Australia (at higher latitudes), and blood collection during winter or spring. We were not able to compare country of origin/race between the AusDiab study and the AHS due to differences in categorisation between the two studies. The AusDiab study reported higher vitamin $\mathrm{D}$ deficiency in people of non-European origin, while we found a higher prevalence of vitamin D deficiency in those born in a country other than Australia or the main Englishspeaking countries. 


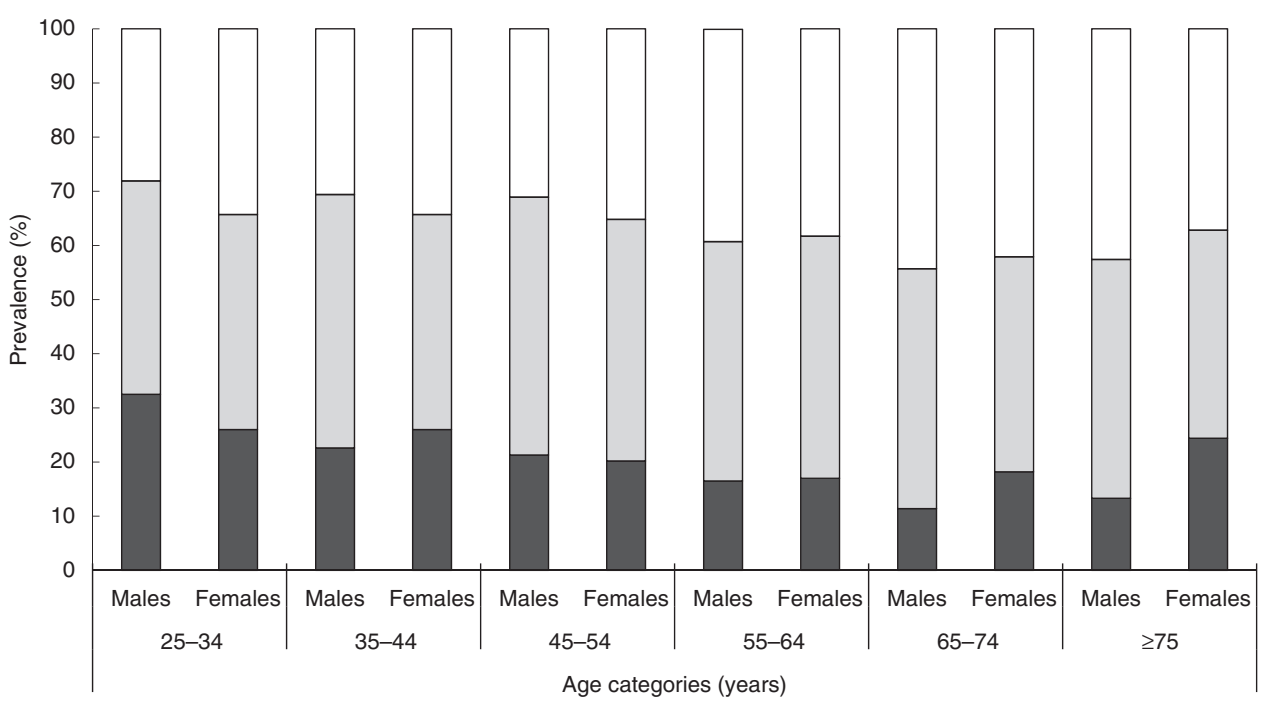

Fig. 1. Prevalence of vitamin $D$ deficiency (serum 25 -hydroxyvitamin $D$ concentrations $<50 \mathrm{nmol} / /(\square)$ ), insufficiency $(50$ to $<75 \mathrm{nmol} / /, \square)$ and sufficiency $(\geq 75 \mathrm{nmol} / /$, $\square$ ) by age group in (a) men ( $n$ 2267) and (b) women ( $n$ 2767) who participated in the 2011-2013 National Health Survey and National Health Measures Survey (weighted to the Australian population in 2011-2013).

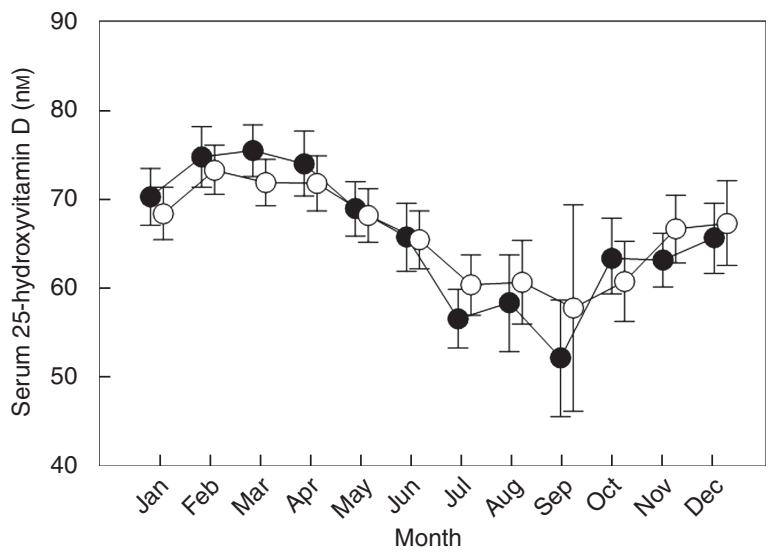

Fig. 2. Mean adjusted serum 25-hydroxyvitamin D concentrations (with $95 \%$ $\mathrm{Cl}$ ) in men and women aged $\geq 25$ years who participated in the $2011-2013$ National Health Survey and National Health Measures Survey ( $n 2267$ men, 2767 women), by month of the year (spring (September-November), summer (December-February), autumn (March-May), winter (June-August)). Mean adjusted for age group, region of birth, state/territory, remoteness area, BMI, smoking, education, physical activity, socio-economic status, vitamin $D$ and/or calcium supplement use.

Likely reasons for the higher prevalence of vitamin D deficiency for those born outside Australia and other main Englishspeaking countries include clothing habits, which reduce sunlight exposure of the skin, and/or darker skin colour, which indicates a higher melanin concentration. Since melanin is a natural sun protector, non-Caucasians require a longer duration of sunlight exposure than their fair-skinned counterparts to synthesise the same amount of vitamin $\mathrm{D}^{(26)}$. Low physical activity may be associated with a decreased amount of time spent outdoors, resulting in decreased cutaneous synthesis of vitamin D. Physical inactivity is also a risk factor for overweight/obesity, and there is an inverse association between body fat and serum 25(OH)D concentrations that may be related to the volumetric dilution of vitamin $\mathrm{D}$ in fat mass $^{(27)}$.
Season and latitude affect cutaneous synthesis of vitamin D: in winter the Earth is further from the sun resulting in lower UV intensity; at higher latitudes, the sun is lower in the sky and UV radiation traverses a longer path through the atmosphere, resulting in greater absorption of UV-B radiation by stratospheric Ozone $^{(28)}$. In addition, cloud cover impacts surface UV-B levels: vitamin D synthesis is reduced in cloudy conditions compared with clear sky ${ }^{(29)}$, which may partially explain differences in the prevalence of vitamin D deficiency between states/territories. In our study, Victoria (the southern-most state of the Australian mainland) had the highest prevalence of vitamin D deficiency. Victoria was also reported as having the highest incidence $(13 / 100000$ per year) of rickets in children aged $\leq 15$ years by the Australian Paediatric Surveillance Unit Study ${ }^{(30)}$. Conversely, the second highest incidence of rickets was seen in Western Australia $^{(30)}$, which ranks sixth of the eight states and territories in terms of prevalence of vitamin D deficiency. Although the prevalence of vitamin D deficiency was high across the more southerly states (Victoria, Tasmania, South Australia), the prevalence of vitamin D deficiency was also high in the Northern Territory, which had the highest prevalence of severe vitamin D deficiency. There is a higher proportion of Aboriginal and Torres Strait Islander people in the Northern Territory compared to other states ${ }^{(31)}$ and, withstanding Darwin city, which the Accessibility and Remoteness Index Australia 2006 classified as outer regional, the remainder of the territory is classified as remote or very remote ${ }^{(32)}$. The Australian Aboriginal and Torres Strait Islander Health Survey 2012-2013 found that the prevalence of vitamin D deficiency in people living in remote areas was nearly $40 \%$, compared with $23 \%$ in non-remote areas ${ }^{(33)}$.

There were differences between the AusDiab study and the AHS with respect to age and sex as predictors of vitamin D deficiency. Results from the AusDiab study showed that vitamin D deficiency was most prevalent in older age groups and women, while our study showed that vitamin D deficiency was highest in the youngest age group (25-34 year olds), with no sex 
Table 4. Unadjusted and adjusted survey-weighted logistic regression models investigating predictors of vitamin D deficiency (serum 25-hydroxyvitamin D concentrations $<50 \mathrm{nmol} / \mathrm{l}$ ) in Australian adults aged $\geq 25$ years included in the present study, stratified by sex (men, $n$ 2267; women, $n$ 2767)

(Odds ratios and $95 \%$ confidence intervals)

\begin{tabular}{|c|c|c|c|c|c|c|c|c|}
\hline \multirow[b]{2}{*}{ Characteristics } & \multicolumn{4}{|c|}{ Men } & \multicolumn{4}{|c|}{ Women } \\
\hline & Unadjusted & $95 \% \mathrm{Cl}$ & Adjusted $\mathrm{C}$ & $95 \% \mathrm{Cl}$ & Unadjusted & $95 \% \mathrm{Cl}$ & Adjusted $\mathrm{O}$ & $95 \% \mathrm{Cl}$ \\
\hline \multicolumn{9}{|l|}{ Age categories (years) } \\
\hline $25-34$ & \multicolumn{2}{|c|}{ Ref. } & \multicolumn{2}{|c|}{ Ref. } & \multicolumn{2}{|c|}{ Ref. } & \multicolumn{2}{|c|}{ Ref. } \\
\hline $35-44$ & 0.61 & $0.40,0.93$ & 0.67 & $0.42,1.09$ & 0.87 & $0.59,1.27$ & 0.98 & $0.65,1.49$ \\
\hline $45-54$ & 0.57 & $0.38,0.85$ & 0.68 & $0.42,1 \cdot 10$ & 0.72 & $0.49,1.04$ & 0.81 & $0.54,1.21$ \\
\hline $55-64$ & 0.41 & $0.27,0.62$ & 0.56 & $0.34,0.90$ & 0.58 & $0.40,0.85$ & 0.72 & $0.46,1.11$ \\
\hline $65-74$ & 0.27 & $0.17,0.43$ & 0.36 & $0.21,0.64$ & 0.63 & $0.42,0.94$ & 0.74 & $0.46,1.19$ \\
\hline$\geq 75$ & 0.32 & $0.19,0.53$ & 0.53 & $0.29,0.96$ & 0.92 & $0.58,1.44$ & $1 \cdot 22$ & $0.70,2.11$ \\
\hline \multicolumn{9}{|l|}{ Region of birth } \\
\hline Australia & \multicolumn{2}{|c|}{ Ref. } & \multicolumn{2}{|c|}{ Ref. } & \multicolumn{2}{|c|}{ Ref. } & \multicolumn{2}{|c|}{ Ref. } \\
\hline Main English-speaking countries† & 0.76 & $0.51,1.15$ & 0.73 & $0.46,1.16$ & $1 \cdot 20$ & $0.85,1.70$ & 1.39 & $0.96,2.01$ \\
\hline Other & $3 \cdot 71$ & $2 \cdot 77,4.96$ & $3 \cdot 18$ & $2 \cdot 28,4.45$ & $2 \cdot 86$ & $2 \cdot 19,3 \cdot 75$ & $3 \cdot 30$ & $2.41,4.51$ \\
\hline \multicolumn{9}{|l|}{ State/territory } \\
\hline New South Wales & \multicolumn{2}{|c|}{ Ref. } & \multicolumn{2}{|c|}{ Ref. } & \multicolumn{2}{|c|}{ Ref. } & & \\
\hline Victoria & 1.87 & $1 \cdot 31,2 \cdot 66$ & 1.74 & $1 \cdot 17,2 \cdot 60$ & 1.45 & $1 \cdot 06,2 \cdot 01$ & 1.66 & $1.17,2.37$ \\
\hline Queensland & 0.34 & $0.22,0.54$ & 0.34 & $0.21,0.56$ & 0.54 & $0.38,0.79$ & 0.52 & $0.35,0.79$ \\
\hline South Australia & 1.42 & $0.97,2.09$ & 1.37 & $0.90,2.09$ & $1 \cdot 23$ & $0.88,1.73$ & 1.39 & $0.95,2.03$ \\
\hline Western Australia & 0.66 & $0.44,0.99$ & 0.64 & $0.41,0.99$ & 0.82 & $0.58,1.73$ & 0.76 & $0.51,1.12$ \\
\hline Tasmania & 1.67 & $1 \cdot 12,2 \cdot 50$ & $4 \cdot 19$ & $2 \cdot 35,7.48$ & $1 \cdot 23$ & $0.86,1.75$ & 2.48 & $1.57,3.91$ \\
\hline Australian Capital Territory & 0.28 & $0.13,0.60$ & 0.54 & $0.20,1.48$ & 0.51 & $0.27,0.98$ & 0.99 & $0.44,2 \cdot 21$ \\
\hline Northern Territory & $1 \cdot 26$ & $0.83,1.93$ & 1.03 & $0.64,1.67$ & 1.45 & $0.92,2.26$ & 1.40 & $0.91,2.15$ \\
\hline Season & & & & & & & & \\
\hline Summer (December-February) & & & & & & & & \\
\hline Autumn (March-May) & 0.87 & $0.60,1.28$ & 0.82 & $0.54,1.26$ & 1.09 & $0.79,1.52$ & 1.07 & $0.75,1.52$ \\
\hline Winter (June-August) & 3.08 & $2 \cdot 16,4 \cdot 39$ & 3.55 & $2 \cdot 39,5 \cdot 27$ & 2.65 & $1.91,3.68$ & 3.36 & $2 \cdot 32,4.86$ \\
\hline Spring (September-November) & 2.08 & $1 \cdot 39,3 \cdot 11$ & $2 \cdot 10$ & $1 \cdot 34,3 \cdot 30$ & 2.54 & $1 \cdot 78,3.62$ & 2.98 & $2 \cdot 03,4.38$ \\
\hline Remoteness areał & & & & & & & & \\
\hline Major cities & & & & & & & & \\
\hline Inner regional & 0.34 & $0.24,0.48$ & 0.38 & $0.24,0.61$ & 0.63 & $0.47,0.85$ & 0.64 & $0.45,0.93$ \\
\hline Other & 0.34 & $0.21,0.54$ & 0.43 & $0.23,0.80$ & 0.47 & $0.33,0.67$ & 0.46 & $0.30,0.72$ \\
\hline BMI§ & & & & & & & & \\
\hline Healthy weight & & & & & & & & \\
\hline Overweight & 0.89 & $0.65,1.23$ & $1 \cdot 11$ & $0.77,1.60$ & 0.83 & $0.62,1.10$ & 0.84 & $0.62,1.15$ \\
\hline Obese & 1.08 & $0.78,1.51$ & 1.53 & $1 \cdot 05,2 \cdot 23$ & 1.64 & $1 \cdot 27,2 \cdot 13$ & 1.97 & $1.45,2.67$ \\
\hline Smoking & & & & & & & & \\
\hline Ex-/non-smoker & & & & & & & & \\
\hline Current & 1.48 & $1.05,2.09$ & 1.41 & $0.94,2.11$ & 1.41 & $1.04,1.91$ & 1.47 & $1.03,2.09$ \\
\hline Education & & & & & & & & \\
\hline High/primary/no school & & & & & & & & \\
\hline Certificate/diploma & 0.87 & $0.64,1.18$ & 0.80 & $0.57,1.14$ & 0.82 & $0.63,1.07$ & 0.83 & $0.61,1.14$ \\
\hline University & 1.84 & $1.34,2.52$ & 1.52 & $1.01,2.29$ & $1 \cdot 24$ & $0.95,1.61$ & $1 \cdot 28$ & $0.92,1.79$ \\
\hline Physical activity\|l & & & & & & & & \\
\hline High & & & & & & & & \\
\hline Moderate & $1 \cdot 10$ & $0.70,1.73$ & 1.47 & $0.86,2.45$ & 1.35 & $0.80,2.26$ & $1 \cdot 38$ & $0.81,2.35$ \\
\hline Low/sedentary & 1.40 & $0.92,2.12$ & 1.98 & $1 \cdot 20,3 \cdot 27$ & 2.45 & $1.51,3.99$ & $2 \cdot 37$ & $1.43,3.94$ \\
\hline Socio-economic status & & & & & & & & \\
\hline Lowest quintile & & & & & & & & \\
\hline Second quintile & 1.08 & $0.72,1.62$ & 0.97 & $0.61,1.53$ & 0.86 & $0.61,1.22$ & 1.05 & $0.71,1.57$ \\
\hline Third quintile & 0.86 & $0.57,1.30$ & 0.79 & $0.49,1.28$ & 0.86 & $0.61,1.21$ & 1.07 & $0.73,1.58$ \\
\hline Fourth quintile & $1 \cdot 23$ & $0.82,1.82$ & 1.03 & $0.65,1.64$ & 0.66 & $0.46,0.94$ & 0.66 & $0.44,0.99$ \\
\hline Highest quintile & $1 \cdot 28$ & $0.86,1.92$ & 0.97 & $0.60,1.57$ & 0.76 & $0.53,1.09$ & 0.88 & $0.58,1.35$ \\
\hline Vitamin D or Ca supplement use & & & & & & & & \\
\hline No & & & & & & & & \\
\hline Yes & 0.35 & $0.16,0.78$ & $0 \cdot 16$ & $0.07,0.40$ & 0.24 & $0.16,0.37$ & 0.16 & $0.09,0.27$ \\
\hline
\end{tabular}

Ref., reference; CD, collection districts.

${ }^{*}$ Adjusted for all other variables.

† Main English-speaking countries: USA, Canada, UK, Republic of Ireland, New Zealand and South Africa.

¥ Categorised according to the Accessibility/Remoteness Index of Australia, which is mapped to the census CD from the Census of Population and Housing (major cities: CD with index values 0-0.20; inner regional: 0.21-2.40; other: 2.41-5.92).

$\S$ Healthy weight $\left(18.5-24.9 \mathrm{~kg} / \mathrm{m}^{2}\right.$; this group includes a small number of underweight participants $\left.<18.5 \mathrm{~kg} / \mathrm{m}^{2}\right)$, overweight $\left(25 \cdot 0-29 \cdot 9 \mathrm{~kg} / \mathrm{m}^{2}\right)$ and obese $\left(\geq 30.0 \mathrm{~kg} / \mathrm{m}^{2}\right)$

II Based on the level of physical activity performed for fitness, sport or recreation and walking for transport in the past week.

I Based on 2011 Index of Relative Socioeconomic Disadvantage. 
differences. These changes may partly be due to an increasing use of vitamin D supplements, particularly in older women. Although the prevalence of single vitamin D supplement use in Australia is generally low, it is higher in older adults than in younger adults and higher in women than men ${ }^{(34)}$. Increasing use of vitamin D supplements over the last 20 years has been shown in the USA from serial NHANES data ${ }^{(35)}$. In addition, a Western Australian Study of 2076 adults aged 18-45 years found that while men were more likely to wear a hat and protective clothing when outdoors, women were more likely to use sunscreen and spend more time in indoor or shaded areas ${ }^{(36)}$, behaviours which may lead to men and women receiving similar amounts of vitamin D from sunlight.

Strengths of our study include the use of a nationally representative sample of the Australian adult population (aged $\geq 25$ years), and the use of an LC-MS/MS method certified to the RMP for measuring serum $25(\mathrm{OH}) \mathrm{D}$ concentrations. Due to the comprehensive data available from the AHS, we were also able to investigate a number of potential predictors of vitamin $\mathrm{D}$ deficiency. However, we were not able to consider the effects of diet or sun exposure, a limitation common to many population-based studies of vitamin D deficiency ${ }^{(9)}$. Due to the differences in assay methodology, we were unable to compare the absolute prevalence of vitamin D deficiency with previous estimates from the AusDiab study. However, while the absolute prevalence may not be comparable due to expected analytical method bias, that bias is not expected to interact with the various predictors; exposure-outcome relationships have been shown to be conserved, despite bias in the prevalence of the outcome $^{(37)}$. Hence, we compared predictors of vitamin D deficiency from the AHS with those from the AusDiab study. A limitation of this comparison is that not all variables were categorised the same way in the two studies. For example, physical activity was derived differently, and some categories were not comparable (e.g. race/ethnicity in the AusDiab study $v$. region of birth in the AHS; location of residence (urban/ rural) in the AusDiab study $v$. remoteness area in the AHS). Furthermore, some variables were not collected in both studies (e.g. television viewing time was collected in the AusDiab study but not in the AHS; supplement use was collected in the AHS but not in the AusDiab study).

\section{Conclusion}

The 2011-2013 AHS provides the first estimate of the prevalence of vitamin $\mathrm{D}$ deficiency in a nationally representative sample of adults using a gold standard method for measuring serum 25(OH)D concentrations. Among Australian adults aged $\geq 25$ years, the prevalence of deficiency $(<50 \mathrm{nmol} / \mathrm{l})$ and insufficiency ( 50 to $<75 \mathrm{nmol} / \mathrm{l}$ ) was 20 and $43 \%$, respectively, while the remaining $37 \%$ of the population were vitamin D sufficient $(\geq 75 \mathrm{nmol} / \mathrm{l})$. The predictors of vitamin D deficiency remained largely the same between the 1999-2000 AusDiab study and the 2011-2013 AHS. However, while age continues to be a major risk factor for vitamin D deficiency, the risk has shifted to a younger age group compared with the AusDiab study, with 25- to 34-year-olds now carrying the highest risk of deficiency. Given our increasingly indoor lifestyles, there is a need to develop and promote strategies to maintain adequate vitamin D status through safe sun exposure and dietary approaches.

\section{Acknowledgements}

The Australian Health Survey was conducted by the Australian Bureau of Statistics (ABS) with funding provided through the ABS health survey programme, the Department of Health and the National Heart Foundation of Australia. We thank the Australian Health Survey participants for their time and contribution to the survey. We gratefully acknowledge Paul Atyeo and Barry Tynan (ABS) for their advice and assistance in using data from the Australian Health Survey.

R. M. L. is supported by an Australian National Health and Medical Research Council Senior Research Fellowship. L. J. B. is supported by a Multiple Sclerosis Western Australia Research Fellowship and a Curtin University Research Fellowship.

R. M. L. and L. J. B. designed the study; E. M. and P. C. analysed and interpreted the data; E. M., P. C. and E. D. wrote the paper; L. J. B., J. L. S., R. M. L., R. M. D. and C. A. N. provided critical revision of the manuscript for important intellectual content; L. J. B. had primary responsibility for the final content. All the authors read and approved the final version of the manuscript.

The authors declare no conflicts of interest.

\section{References}

1. Liu X, Baylin A \& Levy PD (2018) Vitamin D deficiency and insufficiency among US adults: prevalence, predictors and clinical implications. Br J Nutr 119, 928-936.

2. Rockell JE, Skeaff CM, Williams SM, et al. (2006) Serum 25-hydroxyvitamin D concentrations of New Zealanders aged 15 years and older. Osteoporos Int 17, 1382-1389.

3. Sarafin K, Durazo-Arvizu R, Tian L, et al. (2015) Standardizing 25-hydroxyvitamin $\mathrm{D}$ values from the Canadian Health Measures Survey. Am J Clin Nutr 102, 1044-1050.

4. Cashman KD, Dowling KG, Škrabáková Z, et al. (2016) Vitamin D deficiency in Europe: pandemic? Am J Clin Nutr 103, 1033-1044

5. Black LJ, Walton J, Flynn A, et al. (2015) Small increments in vitamin $D$ intake by Irish adults over a decade show that strategic initiatives to fortify the food supply are needed. J Nutr 145, 969-976.

6. Vatanparast H, Calvo MS, Green TJ, et al. (2010) Despite mandatory fortification of staple foods, vitamin D intakes of Canadian children and adults are inadequate. J Steroid Biochem Mol Biol 121, 301-303.

7. Bailey RL, Dodd KW, Goldman JA, et al. (2010) Estimation of total usual calcium and vitamin D intakes in the United States. J Nutr 140, 817-822.

8. Scientific Advisory Committee on Nutrition (2016) Vitamin D and Health. London: UK Government. https://assets.publishing. service.gov.uk/government/uploads/system/uploads/attachment_ data/file/537616/SACN_Vitamin_D_and_Health_report.pdf (accessed February 2019).

9. Daly RM, Gagnon C, ZX Lu, et al. (2012) Prevalence of vitamin D deficiency and its determinants in Australian adults aged 25 years and older: a national, population-based study. Clin Endocrinol 77, 26-35.

10. Sempos CT, Vesper HW, Phinney KW, et al. (2012) Vitamin D status as an international issue: national surveys and the problem of standardization. Scand J Clin Lab Invest 243, 32-40. 
11. Mineva EM, Schleicher RL, Chaudhary-Webb M, et al. (2015) A candidate reference measurement procedure for quantifying serum concentrations of 25-hydroxyvitamin $\mathrm{D}(3)$ and 25 hydroxyvitamin D(2) using isotope-dilution liquid chromatographytandem mass spectrometry. Anal Bioanal Chem 407, 5615-5624.

12. Lai JKC, Lucas RM, Banks E, et al. (2012) Variability in vitamin D assays impairs clinical assessment of vitamin D status. Intern Med J 42, 43-50.

13. Black LJ, Anderson D, Clarke MW, et al. (2015) Analytical bias in the measurement of serum 25-hydroxyvitamin D concentrations impairs assessment of vitamin D status in clinical and research settings. PLOS ONE 10, e0135478.

14. Binkley N, Krueger DC, Morgan S, et al. (2010) Current status of clinical 25-hydroxyvitamin D measurement: an assessment of between-laboratory agreement. Clin Chim Acta 411, 1976-1982.

15. Lucas R \& Neale R (2014) What is the optimal level of vitamin D?: Separating the evidence from the rhetoric. Aust Fam Physician 43, 119-122.

16. Australian Bureau of Statistics (2014) Australian Health Survey: biomedical results for nutrients. http://www.abs.gov.au/ausstats/ abs@.nsf/Lookup/4364.0.55.006main+features12011-12 (accessed March 2018).

17. Australian Bureau of Statistics (2013) Australian Health Survey: users' guide, 2011-2013. http://www.abs.gov.au/ausstats/ abs@.nsf/mf/4363.0.55.001 (accessed March 2018).

18. Australian Bureau of Statistics (2013) Australian Health Survey, National Health Survey, 2011-13, expanded confidentialised unit record file (CURF): ABS DataLab. Findings based on use of ABS microdata. http://www.abs.gov.au/ausstats/abs@.nsf/ $\mathrm{mf} / 4363.0 .55 .001$ (accessed March 2018).

19. Holick MF, Binkley NC, Bischoff-Ferrari HA, et al. (2011) Evaluation, treatment, and prevention of vitamin D deficiency: an endocrine society clinical practice guideline. J Clin Endocrinol Metab 96, 1911-1930.

20. World Health Organization (2016) Obesity and overweight. http://www.who.int/mediacentre/factsheets/fs311/en/ (accessed March 2018).

21. Australian Bureau of Statistics (2017) Census: 2016 data in pictures. http://www.censusdata.abs.gov.au/CensusOutput/ copsub2016.nsf/All\%20docs\%20by\%20catNo/Data-in-pictures/ \$FILE/australiaER.html (accessed February 2019).

22. Centers for Disease Control and Prevention (2009) National Health and Nutrition Examination Survey 2005-2006: documentation, codebook, and frequencies vitamin D. https:// www.cdc.gov/nchs/data/nhanes/nhanes_05_06/vid_d.pdf (accessed June 2018)

23. Looker AC, Pfeiffer CM, Lacher DA, et al. (2008) Serum 25hydroxyvitamin D status of the US population: 1988-1994 compared with 2000-2004. Am J Clin Nutr 88, 1519-1527.

24. Schleicher RL, Sternberg MR, Lacher DA, et al. (2016) The vitamin D status of the US population from 1988 to 2010 using standardized serum concentrations of 25-hydroxyvitamin D shows recent modest increases. Am J Clin Nutr 104, 454-461.

25. Cashman KD, Kiely M, Kinsella M, et al. (2013) Evaluation of vitamin $\mathrm{D}$ standardization program protocols for standardizing serum 25-hydroxyvitamin D data: a case study of the program's potential for national nutrition and health surveys. $\mathrm{AmJ}$ Clin Nutr 97, 1235-1242.

26. Holick MF (2004) Sunlight and vitamin D for bone health and prevention of autoimmune diseases, cancers, and cardiovascular disease. Am J Clin Nutr 80, Suppl., 1678S-1688S.

27. Drincic AT, Armas LA, Van Diest EE, et al. (2012) Volumetric dilution, rather than sequestration best explains the low vitamin D status of obesity. Obesity (Silver Spring) 20, 1444-1448.

28. Holick MF (2004) Vitamin D: importance in the prevention of cancers, type 1 diabetes, heart disease, and osteoporosis. Am J Clin Nutr 79, 362-371.

29. Kimlin MG (2008) Geographic location and vitamin D synthesis. Mol Aspects Med 29, 453-461.

30. Munns CF, Simm PJ, Rodda CP, et al. (2012) Incidence of vitamin D deficiency rickets among Australian children: an Australian Paediatric Surveillance Unit Study. Med J Aust 196, 466-468.

31. Australian Bureau of Statistics (2017) Census: aboriginal and Torres Strait Islander population. http://www.abs.gov.au/ ausstats/abs@.nsf/MediaRealesesByCatalogue/02D50FAA998 7D6B7CA25814800087E03?OpenDocument. (accessed February 2019)

32. Australian Institute of Family Studies (2018) Map of Australia showing areas of varying geographic remoteness. https://aifs. gov.au/publications/families-regional-rural-and-remote-australia/ figure1 (accessed February 2019).

33. Australian Bureau of Statistics (2014) 4727.0.55.003 - Australian aboriginal and Torres Strait Islander Health Survey: biomedical results, 2012-13: vitamin D. http://www.abs.gov. au/ausstats/abs@.nsf/Lookup/by\%20Subject/4727.0.55.003 201213 Main\%20Features Vitamin\%20D 133 (accessed February 2019).

34. Black L, Jacoby P, Nowson C, et al. (2016) Predictors of vitamin D-containing supplement use in the Australian population and associations between dose and serum 25-hydroxyvitamin D concentrations. Nutrients $\mathbf{8}$, E356.

35. Kantor ED, Rehm CD, Du M, et al. (2016) Trends in dietary supplement use among US adults from 1999-2012. JAMA 316, 1464-1474.

36. Koch S, Pettigrew S, Minto C, et al. (2017) Trends in sunprotection behaviour in Australian adults 2007-2012. Australas J Dermatol 58, 111-116.

37. Mealing NM, Banks E, Jorm LR, et al. (2010) Investigation of relative risk estimates from studies of the same population with contrasting response rates and designs. BMC Med Res Methodol 10, 26. 